\title{
A bistatic linear frequency modulated radar for on-the-ground object detection
}

\begin{abstract}
The use of radar systems for detecting on-the-ground objects is a subject of interest for some applications. Among them, foreign object detection systems are important issues in airport aviation safety. Due to the characteristics of the object, a ground-based bistatic radar configuration is introduced in this article. The transmitter sends broadband linear frequency modulated chirp pulses. The reflected pulses are collected simultaneously by at least two ground-based receivers installed in different positions. Accurate range processing is conducted to detect small objects, such as N-type connectors in distances of several meters. A prototype system consisting of one transmitter and two receivers is developed. The system is then launched over land similar in appearance to a runway, and its ability to make an accurate image of the area where the object is placed in different positions is confirmed. Modifications that need to cover a bigger area are also discussed. The system resolution is analyzed and shows that in the case of several existing transmitter-receiver pairs, the best resolution can be achieved by the closer pairs.
\end{abstract}

Keyword: Bistatic; Linear frequency modulated; Radar; Radar imaging 